\title{
Editorial
}

\section{Long-Memory Processes and Applications}

\author{
Yiming Ding, ${ }^{1}$ Yaozhong $\mathrm{Hu},{ }^{2}$ Weilin Xiao, ${ }^{3}$ and Litan Yan ${ }^{4}$ \\ ${ }^{1}$ Wuhan Institute of Physics and Mathematics, Chinese Academy of Sciences, P.O. Box 71010, Wuhan 430071, China \\ ${ }^{2}$ Department of Mathematics, The University of Kansas, Lawrence, KS 66045, USA \\ ${ }^{3}$ School of Management, Zhejiang University, Yuhangtang Road No. 866, Hangzhou 310058, China \\ ${ }^{4}$ Department of Mathematics, Donghua University, 2999 North People's Road, Shanghai 201620, China
}

Correspondence should be addressed to Yaozhong Hu; hu@math.ku.edu

Received 18 May 2014; Accepted 18 May 2014; Published 28 May 2014

Copyright (C) 2014 Yiming Ding et al. This is an open access article distributed under the Creative Commons Attribution License, which permits unrestricted use, distribution, and reproduction in any medium, provided the original work is properly cited.

Long-memory processes have been widely used to describe natural and social phenomena which display long-memory characters. Some important long-memory processes include fractional Brownian motions, bifractional Brownian motions, and some other Gaussian processes. Since they are neither Markov processes nor semimartingales, the beautiful theories of stochastic analysis developed for semimartingale theory or for Markov processes cannot be applied. In the last decades, there has been a large amount of works dedicated to the analysis and application of long-memory processes and the number of research in this and relevant areas continues to grow.

This special issue contains some recent work on this and relevant areas. It consists of 25 papers and covers a wide range of topics, including fractional Brownian motion, subfractional Brownian motions, and fractional Brownian fields. It also includes some related topics such as nonlinear expectation and $G$-Brownian motion.

The first group of papers focuses on fractional Brownian motions and fields. One paper establishes the existence and uniqueness of the global mild solution for stochastic partial differential equation driven by fractional noise and a pure jump Lévy space-time white noise. Another paper considers the problem of pricing equity indexed annuity in fractional Brownian environment. One of the papers deals with the stochastic stability for a hybrid jump-diffusion model by the supplementary variable technique. Another paper studies the stochastic wave equation when the point sources are placed under the influence of fractional Brownian field. The focus is on the identification of the total number of the point sources and the estimation of the location for each point source. One paper constructs ordinary least squares estimators of variogram parameters in long-memory stochastic volatility assuming the discrete observations. The consistency and the asymptotic normality are given. Another paper establishes a relationship between the Lipschitz continuous functions and polar functions, including fractional Brownian motion and the Brownian sheet and many others. The Hausdorff and packing dimensions of the times set for a nonpolar function are obtained. Another paper provides stochastic stability criterions for the stochastic differential equations driven by fractional Brownian motions. One of the papers combines the autocorrelation approach and the maximum likelihood approach to estimate the Hurst parameter in the fractional Brownian motion when the Hurst index is greater than one half. Another paper extends laws of large numbers under upper probability to sequences of stochastic processes generated by linear interpolation. This extension characterizes the relation between sequences of stochastic processes and subsets of continuous function space in the framework of upper probability. Limit results for sequences of functional random variables and some useful inequalities are also obtained as applications. Another paper studies the asymptotic behavior of some functionals of two independent subfractional Brownian motions with different indices. One paper considers a parameter estimation problem in an $\alpha$ fractional bridge model. Another paper obtains an averaging principle for a class of stochastic differential delay equations driven by fractional Brownian motion with Hurst parameter greater than $1 / 2$. Another paper deals with the existence and 
uniqueness of a mixed stochastic Volterra equation driven by Wiener process and fractional Brownian motion with Hurst parameter, $H>1 / 2$.

The second group of papers concerns the relevant areas such as nonlinear expectation, delay equations, and GBrownian motions. One paper investigates the defective renewal equations under the nonconvolution equivalent distribution class. The asymptotics of the solution are given for the heavy-tailed and light-tailed cases. Another paper is concerned with the stability of stochastic delay differential systems with delayed impulses by Razumikhin methods. Some criteria on the $p$ th moment and almost sure exponential stability are obtained. One of the papers studies the meanfield forward-backward doubly stochastic differential equations, which extend many important equations well studied before. Another paper solves the optimal portfolio selection model under the framework of the prospect theory with decision rule replaced by the $g$-expectation. One paper shows the uniqueness of stationary distribution and asymptotic pathwise estimation for Lotka-Volterra population model with jumps. Moreover, some characterizations for stationary distribution are provided. Another paper establishes the nonadditive version of weak law of large numbers for the independent and identically distributed random variables under Choquet expectations induced by 2-alternating capacities. One of the papers is devoted to the stochastic optimal control problems for systems governed by forward-backward stochastic Volterra integral equations with state constraints. Another paper focuses on fully coupled forward-backward stochastic differential equations with jumps and investigates the associated stochastic optimal control problem along with stochastic maximum principle. Another paper considers a class of neutral stochastic partial differential equations with infinite delay in real separable Hilbert spaces and derives the existence and uniqueness of mild solutions under some local Carathéodory-type conditions and exponential stability in mean square of mild solutions as well as its sample paths. One of the papers obtains a representation theorem for the generators of BSDEs driven by G-Brownian motions and some equivalent results for nonlinear expectations generated by $G$-BSDEs. Another one gets the exponential $G$ martingale theorem with the Kazamaki condition. Another paper investigates the tailed asymptotic behavior of the randomly weighted sums with increments with convolutionequivalent distributions.

Yiming Ding Yaozhong $\mathrm{Hu}$

Weilin Xiao

Litan Yan 


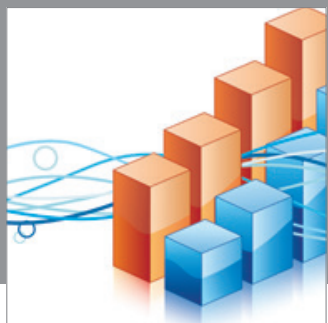

Advances in

Operations Research

mansans

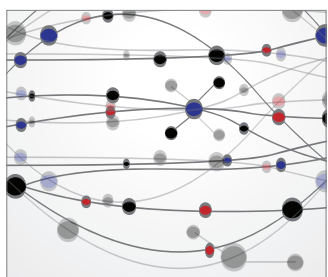

The Scientific World Journal
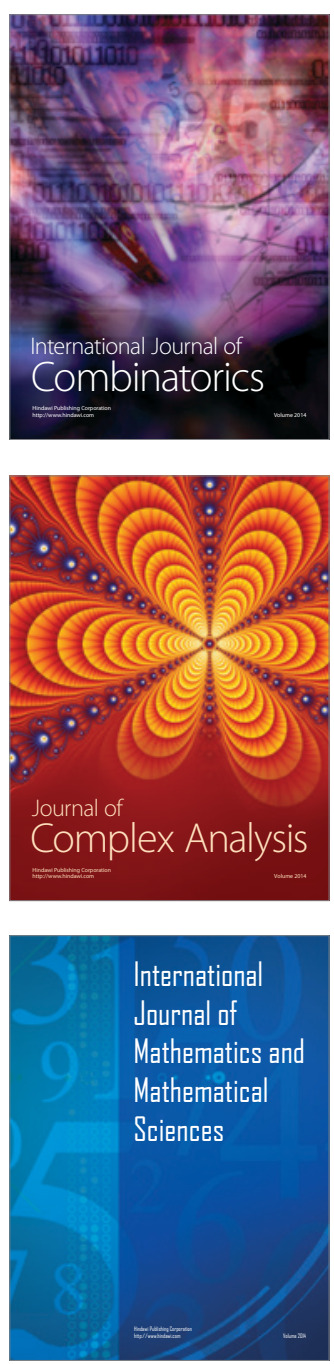
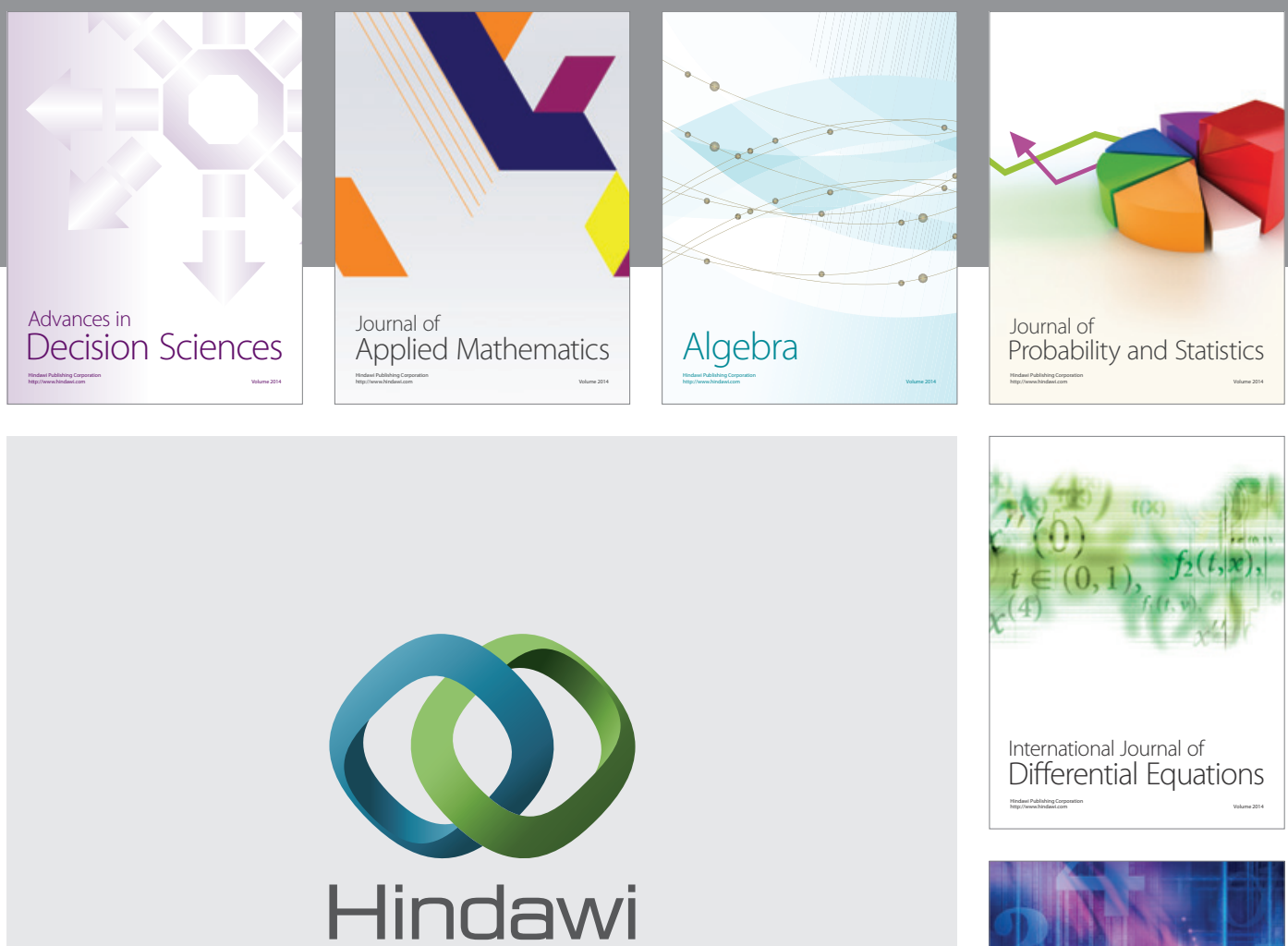

Submit your manuscripts at http://www.hindawi.com
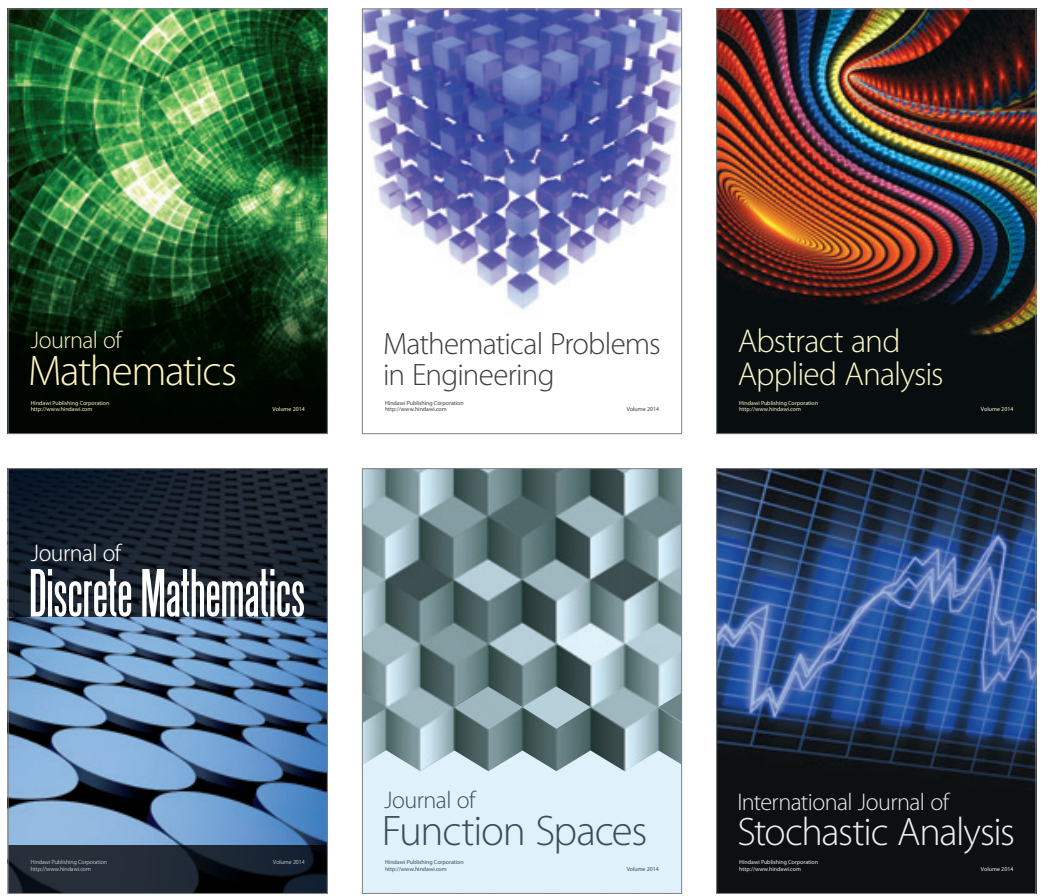

Journal of

Function Spaces

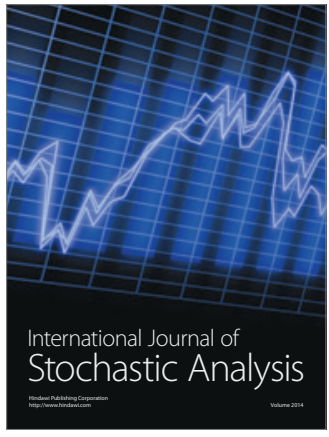

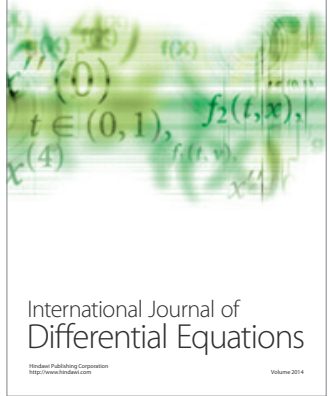
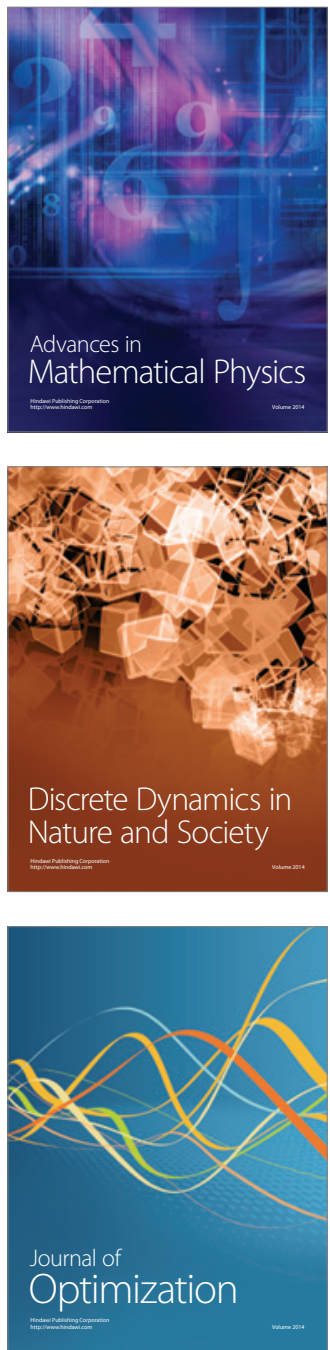